\title{
Autophagy Protects Renal Tubular Cells Against Ischemia / Reperfusion Injury in a Time-Dependent Manner
}

\author{
Xuejing Guan Yingying Qian Yue Shen Lulu Zhang Yi Du Huili Dai Jiaqi Qian \\ Yucheng Yan
}

Department of Nephrology, Molecular Cell Lab for Kidney Disease, Ren Ji Hospital, School of Medicine, Shanghai Jiao Tong University, Shanghai, China

\section{Key Words}

Ischemia/reperfusion • Hypoxia/reoxygenation • Autophagy • Rapamycin • 3-methyladenine

\begin{abstract}
Background/Aims: Autophagy is a dynamic catabolic process that maintains cellular homeostasis. Whether it plays a role in promoting cell survival or cell death in the process of renal ischemia/reperfusion (I/R) remains controversial, partly because renal autophagy is usually examined at a certain time point. Therefore, monitoring of the whole time course of autophagy and apoptosis may help better understand the role of autophagy in renal I/R. Methods: Autophagy and apoptosis were detected after mice were subjected to bilateral renal ischemia followed by 0 -h to 7-day reperfusion, exposure of TCMK-1 cells to $24-h$ hypoxia, and 2 to 24-h reoxygenation. The effect of autophagy on apoptosis was assessed in the presence of autophagy inhibitor 3-methyladenine (3-MA) and autophagy activator rapamycin. Results: Earlier than apoptosis, autophagy increased from 2-h reperfusion, reached the maximum at day 2 , and then began declining from day 3 when renal damage had nearly recovered to normal. Exposure to 24-h hypoxia induced autophagy markedly, but it decreased drastically after 4 and 8-h reoxygenation, which was accompanied with increased cell apoptosis. Inhibition of autophagy with 3-MA increased the apoptosis of renal tubular cells during I/R in vivo and hypoxia/reoxygenation $(H / R)$ in vitro. In contrast, activation of autophagy by rapamycin significantly alleviated renal tissue damage and tubular cell apoptosis in the two models. Conclusion: Autophagy was induced in a time-dependent manner and occurred earlier than the onset of cell apoptosis as an early response that played a renoprotective role during renal $I / R$ and cell $H / R$. Up-regulation of autophagy may prove to be a potential strategy for the treatment of acute kidney injury.
\end{abstract}




\section{Introduction}

Autophagy is a conserved cellular catabolic process that can engulf cytoplasmic components and degrade through the lysosomal pathway to recycle nutrients of the body. In the presence of sufficient carbons, excess peroxisomes can be cleared by autophagy to maintain intracellular homeostasis [1]. Autophagy impairment may be involved in various human disorders such as neurodegeneration diseases, Crohn's disease, pulmonary diseases and kidney diseases [2-5]. Generally, autophagy is thought to be induced under stress conditions $[4,6,7]$. But whether the induction of autophagy contributes to cell survival or cell death remains elusive.

Ischemia/reperfusion (I/R) injury is one of the most common causes of acute kidney injury (AKI). However, the mechanism is not fully understood. In the past few years, a large number of studies have demonstrated that renal I/R injury can induce autophagy. Isaka and his colleagues $[8,9]$ noticed the presence of abundant autophagic vacuoles in tubular epithelial cells in mice after I/R injury, and the number of these autophagic vacuoles increased gradually and reached the peak at 24-h of reperfusion in GFP-LC3 transgenic mice. Jiang et al. [10] also reported the induction of autophagy in renal I/R models in vivo and in vitro. Nevertheless, the time-course of autophagy and the crosstalk between autophagy and apoptosis remain unclear, especially during early I/R injury and late recovery stages in the kidney.

Whether autophagy has a pro-survival or pro-apoptosis function in renal I/R remains controversial. Some studies [11] reported that enhanced autophagy could attenuate apoptosis in I/R induced LLC-PK1 cell injury in vitro, and other studies $[9,12,13]$ found that inhibition of autophagy by renal proximal tubule-specific autophagy associated gene (Atg) knockout sensitized mice to renal I/R injury than their wild-type littermates, thus confirming the renoprotective effect of autophagy. However, some studies $[8,14,15]$ argued that inhibition of autophagy could significantly inhibit $\mathrm{H}_{2} \mathrm{O}_{2}$-induced renal tubular epithelial cell death and attenuate renal I/R injury. In addition, the role of autophagy in in vitro $\mathrm{H} / \mathrm{R}$, mimics in vivo renal I/R also remains unclear. The aim of the present study was to see whether autophagy played a role in apoptosis in a time-dependent manner during in vivo renal I/R and in vitro $\mathrm{H} / \mathrm{R}$ in order to further confirm the association between autophagy and apoptosis. We also intended to see whether autophagy played a renoprotective role using pharmacological inhibitor 3-MA and activator rapamycin, knowing that the understanding about the role of autophagy in renal I/R injury may be able to provide a potential strategy for the treatment of AKI.

\section{Materials and Methods}

Animal experiments

BalB/C male mice aged 7-8 weeks were purchased from Shanghai Slac Animal Laboratory in China. All animal experiments were performed according to a protocol approved by Ren Ji Hospital, School of Medicine, Shanghai Jiao Tong University (Shanghai, China). Mice weighed 20-25 g were subjected to renal I/R operation. Briefly, after anesthesia with intraperitoneal (i.p.) injection of $50 \mathrm{mg} / \mathrm{kg}$ pentobarbital on a heating pad $\left(37^{\circ} \mathrm{C}\right)$, the bilateral renal arteries of the animal were clamped with arterial clips for 35-min before reperfusion for different designated time. In sham operation group, the bilateral renal arteries were exposed for 35-min via a midline abdominal incision without being clamped. Rapamycin (1 mg/kg, Sigma) and 3-MA (30 mg/kg, Sigma) were administered i.p. 2-h before 30-min ischemia for autophagy induction and suppression, respectively. The animals were sacrificed at different time points after reperfusion to collect the blood and kidneys for analysis.

Cell culture

TCMK-1 cells were purchased from American Type Culture Collection (ATCC, CCL-139 ${ }^{\mathrm{TM}}$ ), cultured in Dulbecco Modified Eagle Medium (DMEM, HyClone Technologies) supplemented with 10\% heat-inactivated 


\section{Cellular Physiology Cell Physiol Biochem 2015;36:285-298

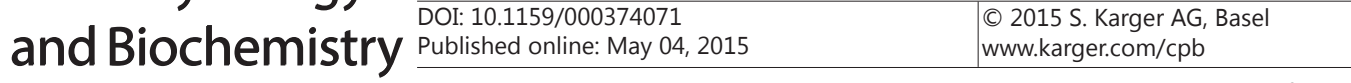 \\ Guan et al.: Autophagy Functions as Renoprotective Role in Renal Ischemia/Reperfusion}

fetal bovine serum (FBS, HyClone Technologies). For hypoxia treatment, TCMK-1 cells were incubated in Forma Series II Water Jackted $\mathrm{CO}_{2}$ Incubator (Thermo) containing $94 \% \mathrm{~N}_{2}, 5 \% \mathrm{CO}_{2}$ to maintain the oxygen concentration at $1 \%$. During reoxygenation incubation, cells were removed to a normoxic chamber $(21 \%$ $\mathrm{O}_{2}$ ). Rapamycin (1 uM, Sigma) and 3-MA (5 mM, Sigma) were administered 4 and 1-h before hypoxia or normoxia for autophagy activation and suppression, respectively. Cells in normoxic group were cultured in the normoxic chamber $\left(21 \% \mathrm{O}_{2}\right)$ at indicated time points.

\section{Electron microscopic examination}

Kidney tissues were harvested immediately after the mice were sacrificed under anesthesia. An approximately $1 \mathrm{~mm}^{3}$ kidney tissue was fixed in $2 \%$ glutaraldehyde. TCMK- 1 cells were grown on a $10 \mathrm{~cm}$ culture dish and fixed in 2\% glutaraldehyde for 4 -h. The kidney tissues and TCMK-1 cells were then postfixed with $2 \%$ Os04, embedded in araldite, stained with uranyl acetate and lead citrate, sliced into ultrathin sections, and observed under an electron microscope (JEM.1010; JEOL, Tokyo, Japan).

Renal function and histologic assay

Renal function was evaluated by monitoring blood urea nitrogen (BUN) and serum creatinine (Scr) with an automatic biochemical analyzer (Hitachi, Japan) at different time points. The kidneys were fixed in $4 \%$ paraformaldehyde, embedded in paraffin, sliced into 4 um sectioned, and stained with hematoxylineosin (HE).

\section{Cell viability}

Cell viability was detected with a Cell Counting Kit-8 (CCK-8, Dojindo) according to the manufacturer's instructions. The absorbance was measured using a microplate reader at a wavelength of $450 \mathrm{~nm}$. The percentage of living cells was calculated by the ratio of absorbance of the experimental group to that of the normoxic group.

\section{Detection of apoptosis by Annexin V-FITC/PI staining and TUNEL assay}

Both the supernatant and adherent cells were collected and incubated with $100 \mu \mathrm{l} 1 \times$ binding buffer containing $5 \mu \mathrm{l}$ Annexin V-FITC and $5 \mu \mathrm{l}$ propidium iodide (PI) (BD). After 30-min incubation in the dark at room temperature, cells were analyzed by flow cytometry (Becton Dickinson FACS Vantage SE, Sanjose). Cells stained with annextin V+/PI- were considered early apoptotic. Apoptotic morphological detection was performed by terminal deoxynucleotidyl transferase dUTP nick end labeling (TUNEL) assay according to the manufacturer's instructions (Roche). TUNEL-positive cells were observed under a fluorescence microscope. Ten representative fields were selected for each group, and the number of TUNEL-positive cells per field of the tissue section or per 500 nuclei in the cell slide was evaluated.

\section{Immunoblot analysis}

Whole cell or tissue lysates were extracted and the protein concentration was determined using a bicinchoninic acid reagent from Pierce (Thermo). An equal amount of protein was electrophoresed in 8-12\% SDS-polyacrylamide gel, and transferred to nitrocellulose membranes (Millipore). The membranes with blotted proteins were blocked for 1 -h with $5 \%$ bovine serum albumin (BSA) at room temperature, and then incubated with anti-LC3 (1:1000, Sigma), anti-p62 (1:500, Proteintech), anti-Bcl-2 (1:1000, Abcam), antiBax (1:1000, Abcam), anti-phosphor-mTOR (1:1000, Cell Signaling Technology), anti-phosphor-P70S6K (1:1000, Cell Signaling Technology), anti-phosphor-4E-BP1 (1:1000, Cell Signaling Technology), anti-mTOR (1:1000, Cell Signaling Technology), anti-P70S6K (1:500, Cell Signaling Technology), anti-4E-BP1 (1:1000, Cell Signaling Technology), and anti-GAPDH (1:1000, Santa cruz) antibodies overnight at $4^{\circ} \mathrm{C}$. After three washes with Tris buffered saline containing 0.05\% Tween-20 (TBST) for 15-min, the membranes were incubated with secondary IRDye 800CW goat anti-rabbit IgG or goat anti-mouse IgG (1:5000; Odyssey) for 1 -h, and then scanned with an infrared imaging system (Odyssey).

\section{Statistics}

Quantitative data were representative of at least three experiments and expressed as means \pm SD. Statistical analysis was performed using the GraphPad Prism software. Statistical differences between groups were analyzed by one-way ANOVA followed by Tukey's post-tests. $p<0.05$ was considered statistically significant. 


\section{Cellular Physiology Cell Physiol Biochem 2015;36:285-298 \\ \begin{tabular}{ll|l} 
and Biochemistry & $\begin{array}{l}\text { DOI: 10.1159/000374071 } \\
\text { Published online: May 04, } 2015\end{array}$ & $\begin{array}{l}\text { C 2015 S. Karger AG, Basel } \\
\text { www.karger.com/cpb }\end{array}$ \\
\hline
\end{tabular}}

\section{Results}

Autophagy occurs before apoptosis and necrosis induced by renal I/R in mice

Autophagy is a dynamic cellular catabolic process accompanied with LC3-II conversion from LC3-I and p62 degradation [16]. Monitoring the time course of autophagy and apoptosis during renal I/R can better understand their relationship. As shown in Fig. 1, A-C, a basal level of autophagy was observed in sham group, but ischemia alone did not induce autophagy, as indicated by the expression of LC3-II/L3-I and p62. Autophagy was induced remarkably 2-h after reperfusion, peaked on day 2 , began declining from day 3 , when it was still higher than that in sham operation group, and finally returned to the baseline level on day 7 . Electron microscopy observed that autophagosomes engulfed dysfunctional mitochondria and other cytoplasmic components when the mice were subjected to 24-h reperfusion (Fig. 1, D). Of note, apoptosis of the renal tissue did not increase until 12-h after reperfusion as indicated by the low level of Bcl-2/Bax (Fig. 1, E-F), reached the maximum on day 1, and then decreased to normal after 3-day reperfusion, suggesting that autophagy could postpone the initiation of the apoptotic program; if autophagy failed to do so, apoptosis would take over [17]. It was observed in this study that autophagy was an early response to renal I/R injury and could postpone the occurrence of apoptosis.

It was also found that Scr and BUN concentrations began increasing 2 and 5-h after reperfusion respectively, reached 9 and 5 fold that of sham operation group at 24-h after reperfusion (Scr: $130.00 \pm 30.07 \mathrm{umol} / \mathrm{L} v s .14 .00 \pm 6.60 \mathrm{umol} / \mathrm{L} ; \mathrm{BUN}$ : $45.57 \pm 8.67 \mathrm{mmol} / \mathrm{L} v s$. $8.18 \pm 2.17 \mathrm{mmol} / \mathrm{L}, p<0.001$ ), began decreasing 2 days after reperfusion, and returned to the normal level 7 and 2 days after reperfusion respectively (Fig. 1, G-H). These results further confirmed the renoprotective function of autophagy.

HE staining showed no significant sign of necrosis of renal tubular epithelial cells before 12-h of reperfusion (data not shown). The renal tissue was damaged obviously 24-h after reperfusion, as indicated by loss of the brush border of proximal tubular epithelial cells, expansion and blockage of the tubular lumen due to cell degeneration, necrosis and shedding, exposure of the basement membrane, and interstitial inflammatory cell infiltration. After 7 days reperfusion, the renal tubular damage was reduced significantly, although vacuolar degeneration was still observed as compared with sham operation group (Fig. 1, I).

\section{Inhibition of autophagy by 3-MA aggravates renal I/R injury}

3-MA is a PI3K inhibitor that can inhibit sequestration of autophagosomes at an early stage resulting in reduced LC3-II/LC3-I and the number of autophagy structures [18], which is always used to clarify the effect of autophagy. As shown by immunoblot assays in our study, 3-MA pretreatment decreased the ratio of LC3-II/LC3-I and increased the expression of p62 significantly at 24-h of reperfusion (Fig. 2, A-C). Meanwhile, the level of Bcl-2/Bax further declined, suggesting that suppression of autophagy by 3-MA aggravated apoptosis of the renal tissue (Fig. 2, A, D). The BUN and Scr concentrations increased to 1.47 and 1.49 fold respectively, as compared with ID1 group (BUN: $66.78 \pm 11.13 \mathrm{mmol} / \mathrm{L} v \mathrm{~s} .45 .57 \pm 10.39$ $\mathrm{mmol} / \mathrm{L}, p<0.05$; Scr: $186.26 \pm 33.75 \mathrm{umol} / \mathrm{L} v s .125 .21 \pm 27.82 \mathrm{umol} / \mathrm{L}, p<0.05$ ) (Fig. 2, E-F). Histological damage confirmed by HE-staining showed more significant dilation of proximal tubules, casts, loss of the brush border and cell necrosis after addition of 3-MA before I/R (Fig. 2 , G). In addition, TUNEL-positive cells increased significantly when 3-MA was administered prior to renal I/R (Fig. 2, H-I). These results indicate that inhibition of autophagy by 3-MA aggravated the renal I/R injury, suggesting that autophagy might play a renoprotective role in renal I/R.

\section{Autophagy activated by rapamycin attenuates renal I/R injury}

Rapamycin is an inhibitor of mammalian target of rapamycin (mTOR) that can activate autophagy both in vitro and in vivo [19]. Immunoblot analysis in our study showed that rapamycin promoted the up-regulation of LC3-II/LC3-I and down-regulation of p62 during renal $I / R$, thus increasing the ratio of Bcl-2/Bax (Fig. 3, A-D). In addition, mTOR 
Fig. 1. Autophagy occurs before apoptosis and necrosis induced by renal $I / R$ in mice. $\mathrm{BalB} / \mathrm{C}$ mice were subjected to sham operation or 35-min ischemia followed by 0 -h to 7-day reperfusion. A: Representative immunoblots of LC3 and p62 in kidney tissue lysates. The blots were reprobed for GAPDH. B and C: Densitometric analysis of LC3 and p62 blots. D: Electron microscopy observed engulfment of dysfunctional mitochondria by autophagosomes (arrows, $x$ 13500) in ID1 group. E: Representative immunoblots of Bcl-2 and Bax in kidney tissue lysates. The blots

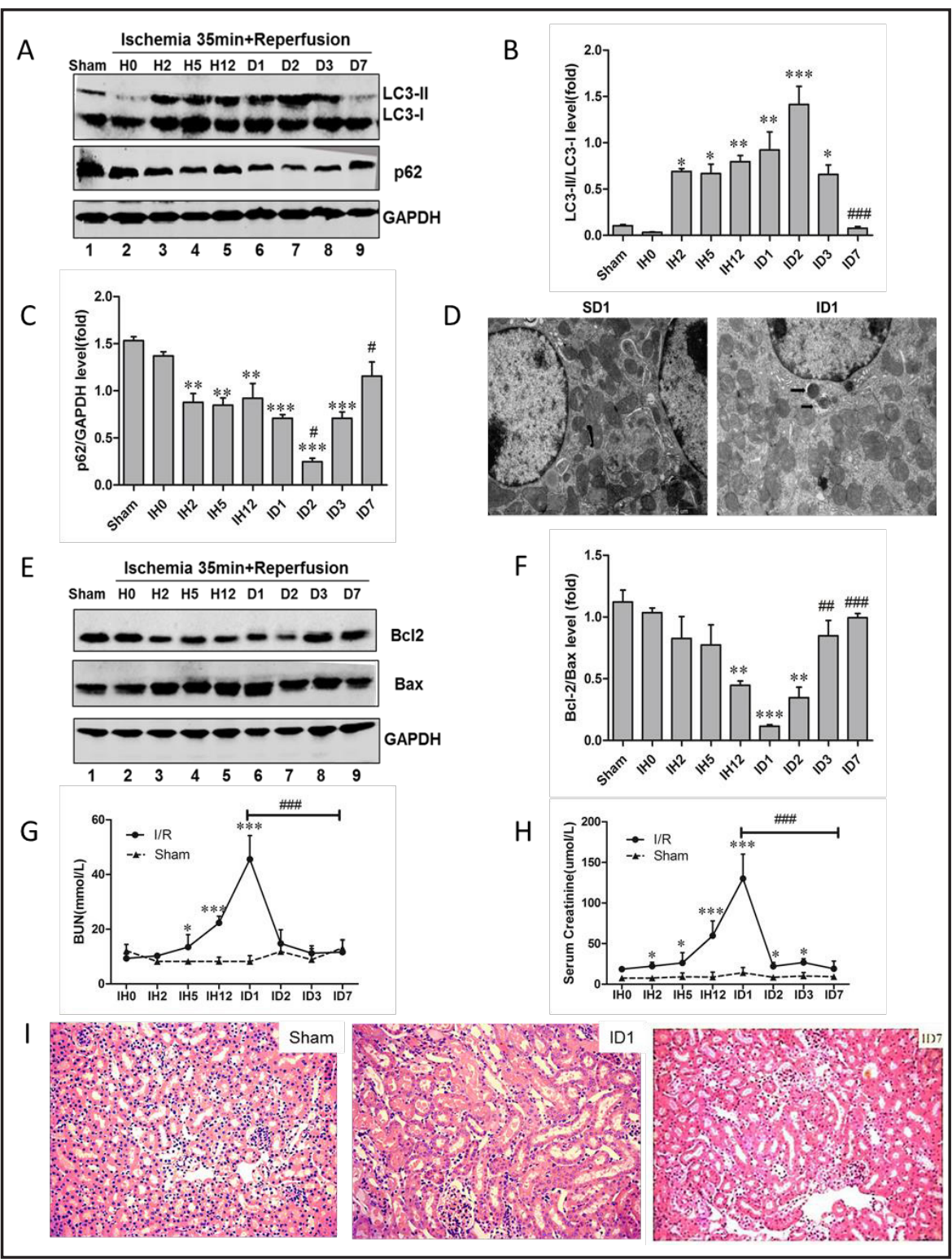

were reprobed for GAPDH. F: Densitometric analysis of Bcl-2/Bax blots. G and H: BUN and Scr concentrations. I: Representative images of renal histology (HE staining, $\times 200$ ). Data are expressed as mean \pm SD (for each group, $\mathrm{n}=3-5$ ). ${ }^{*} p<0.05,{ }^{* *} p<0.01,{ }^{* * *} p<0.001$ significantly different from sham operation group; ${ }^{\#} p<0.05,{ }^{\# \#} p<0.01,{ }^{\# \# \#} p<0.001$ significantly different from ID1 group.

phosphorylation was inhibited in the presence of rapamycin both in sham and I/R groups (Fig. 3, E-F).

The loss of renal function and histological damage were partly but significantly alleviated in the presence of rapamycin, as indicated that the BUN level decreased from $45.57 \mathrm{mmol} / \mathrm{L}$ to $27.3 \mathrm{mmol} / \mathrm{L}$, and Scr decreased from $125.21 \mathrm{umol} / \mathrm{L}$ to $75.36 \mathrm{umol} / \mathrm{L}$ (Fig. 3, G-H). HEstaining also showed dilation of proximal tubules, casts, loss of the brush border and cell necrosis were partly attenuated (Fig. 3, I). Consistent with these results, TUNEL assay showed that the number of apoptotic cells reduced drastically in rapamycin group subjected to renal I/R (Fig. 2, H-I). Of interest, the dose of 3-MA or rapamycin used in sham operation group did not induce significant nephrotoxicity, as evidenced by immunoblot analysis, BUN and Scr concentrations as well as histological examinations. These data suggest that autophagy 


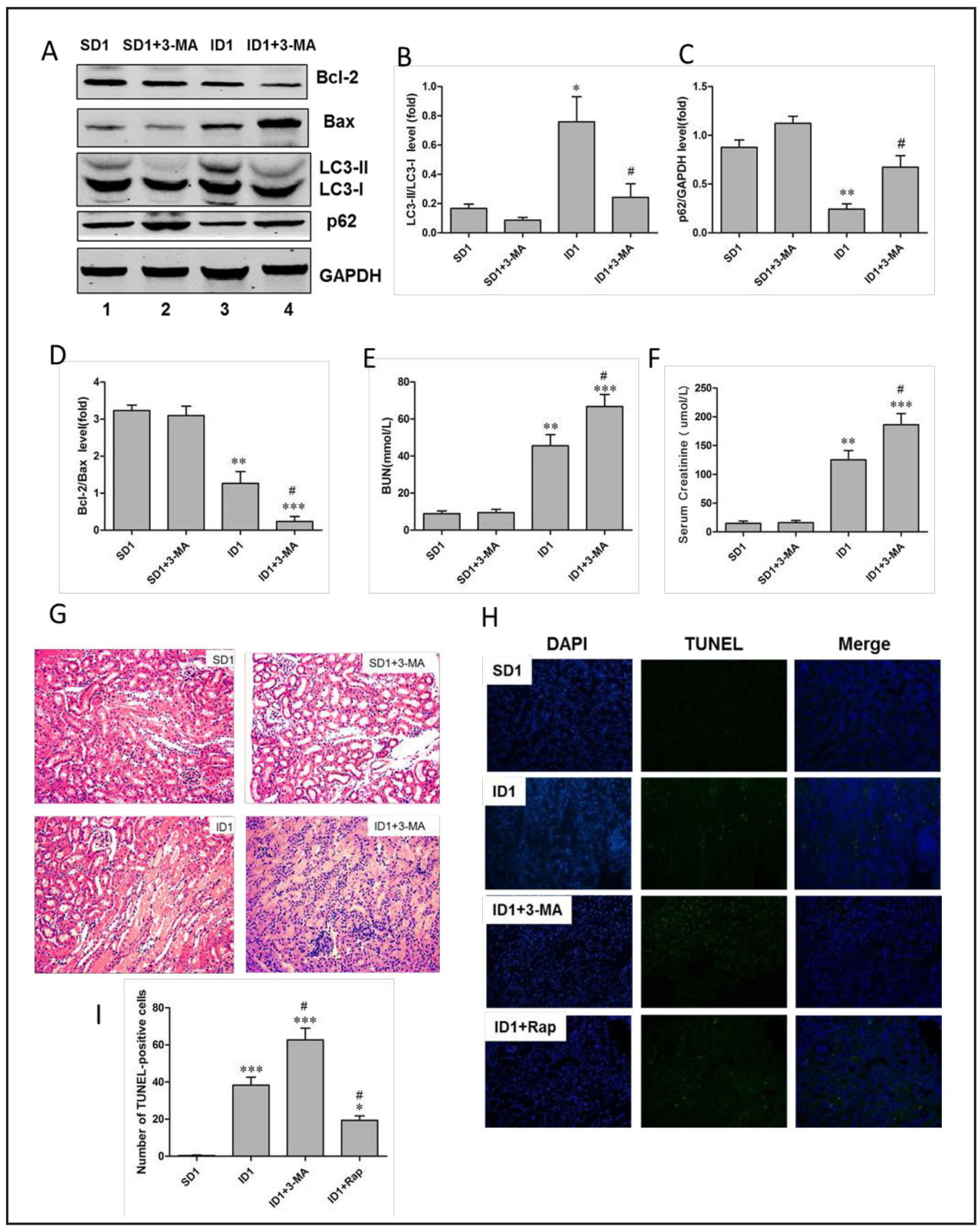

Fig. 2. Inhibition of autophagy by 3-MA aggravates renal I/R injury. 3-MA (30 mg/kg) was injected i.p. 2-h before sham operation or 30-min ischemia followed by 24-h reperfusion. A: Representative immunoblots of LC3, p62, Bcl-2 and Bax in kidney tissue lysates. The blots were reprobed for GAPDH. B-D: Densitometric analysis of LC3, p62, Bcl-2/Bax blots. E and F: BUN and Scr concentrations. G: Representative images of renal histology (HE staining, $\times 200$ ). $\mathrm{H}$ and I: Representative images and numbers of TUNEL-staining cells in kidney tissue $(\times 200)$. Data are expressed as mean \pm SD (for each group, $\mathrm{n}=3-5$ ). ${ }^{*} p<0.05,{ }^{* *} p<0.01,{ }^{* * *} p<0.001$ significantly different from SD1 group; ${ }^{*} p<0.05$, significantly different from ID1 group.

activated by rapamycin attenuated renal I/R injury, and that induction of autophagy in renal I/R may play renoprotective role in I/R settings.

\section{KARGER}




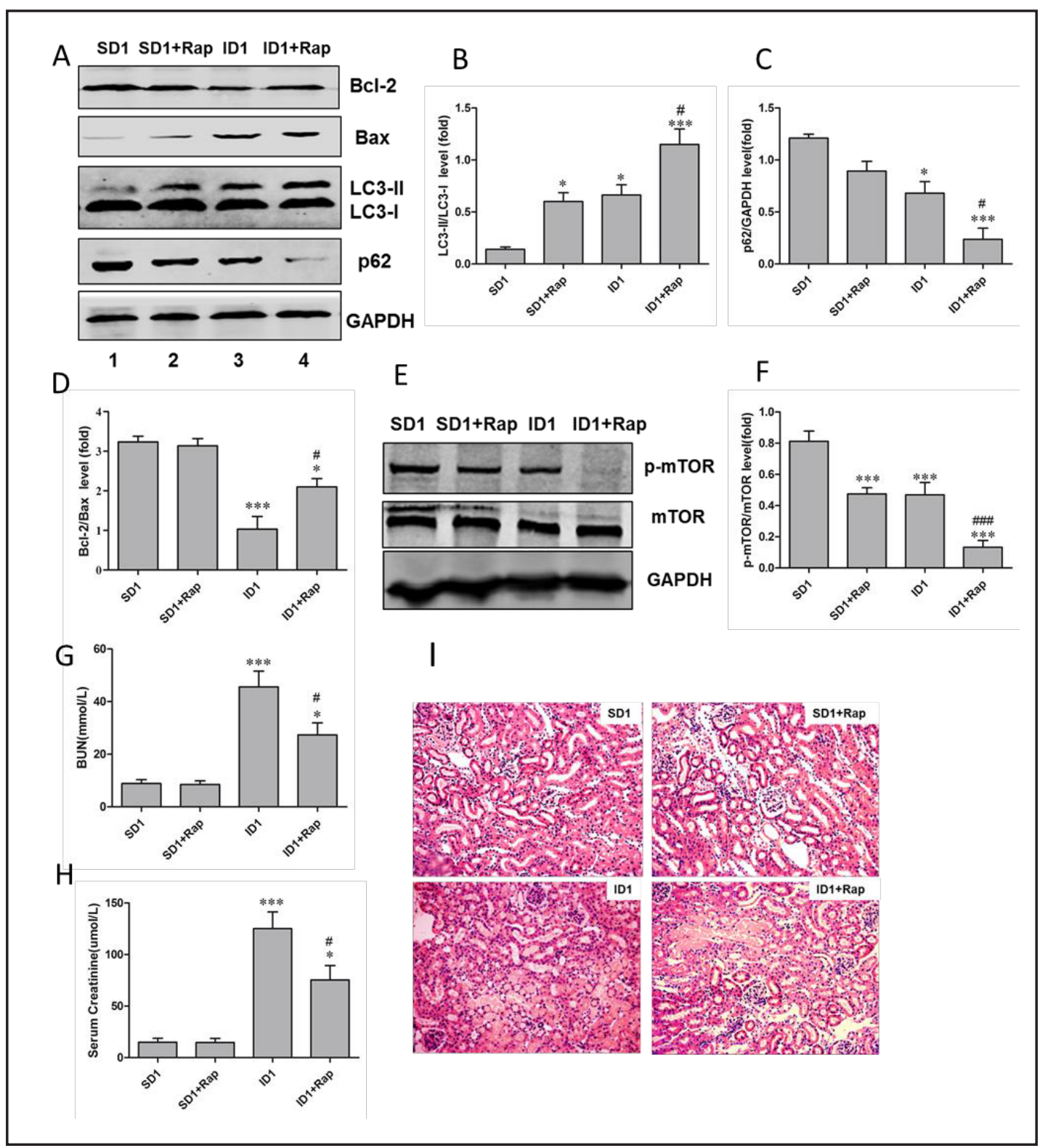

Fig. 3. Autophagy activated by rapamycin attenuates renal I/R injury. Rapamycin (1 mg/kg) was injected i.p. 2-h before sham operation or 30-min ischemia followed by 24-h reperfusion. A: Representative immunoblots of LC3, p62, Bcl-2 and Bax in kidney tissue lysates. The blots were reprobed for GAPDH. B-D: Densitometric analysis of LC3, p62 and Bcl-2/Bax blots. E: Representative immunoblots of p-mTOR in kidney tissue lysates. The blots were reprobed for GAPDH. F: Densitometric analysis of p-mTOR blots. G and H: BUN and Scr concentrations. I: Representative images of renal histology (HE staining, $\times 200$ ). Data are exessed as mean \pm SD (for each group, $\mathrm{n}=3-5$ ). ${ }^{*} p<0.05,{ }^{* *} p<0.01,{ }^{* *} p<0.001$ significantly different from SD1 group; ${ }^{\#} p<0.05$, \#\#\# $p<0.001$ significantly different from ID1 group.

Autophagy induced under hypoxia with or without reoxygenation protects cells against apoptosis

Knowing that renal I/R is often accompanied by hypoxia/reperfusion, we then checked the time course of autophagy and apoptosis in TCMK-1 induced by hypoxia/reoxygenation in vitro. Immunoblot analysis showed a basal level autophagy in TCMK-1 under normoxia, and 6-h hypoxia did not induce autophagy. With prolonged treatment of hypoxia, autophagy was increased significantly at 12 -h and further increased at 24-h. Interestingly, the level of 


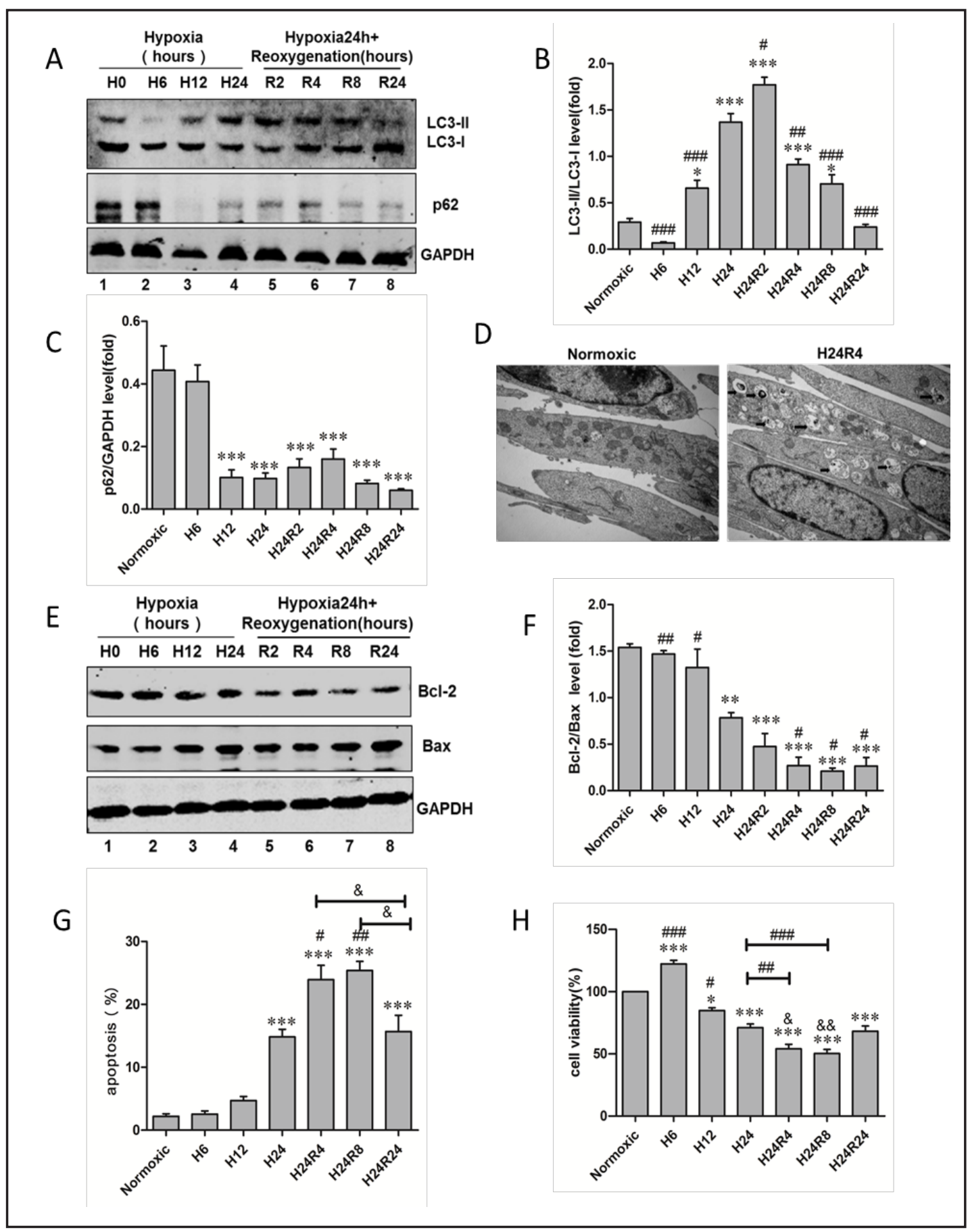

Fig. 4. Autophagy induced under hypoxia with or without reoxygenation protects cells against apoptosis. TCMK-1 was subjected to 0 to 24-h hypoxia alone or 24-h hypoxia followed by 2 to 24-h reoxygenation A: Representative immunoblots of LC3 and p62 in TCMK-1 lysates. The blots were reprobed for GAPDH. B-C: Densitometric analysis of LC3 and p62 blots. D: Electron microscopy observed engulfment of degraded cytoplasmic components by autopagosomes (arrows, $\times 13500$ ) in H24R4 group. E: Representative immunoblots of Bcl-2 and Bax in TCMK-1 lysates. The blots were reprobed for GAPDH. F: Densitometric analysis of Bcl-2/Bax blots. G: Cell apoptosis was detected by Annexin-V/PI staining. H: CCK-8 assay was performed to examine cell viability. Data are expressed as mean \pm SD (for each group, $\mathrm{n}=3$ ) ${ }^{*} p<0.05,{ }^{* *} p<0.01,{ }^{* * *} p<0.001$ significantly different from normoxic group; ${ }^{*} p<0.05$, ${ }^{\# \#} p<0.01$, \#\#\# $p<0.001$ significantly different from H24 group. ${ }^{\&} p<0.05$, \&\& $p<0.01$ significantly different from H24R24 group.

\section{KARGER}




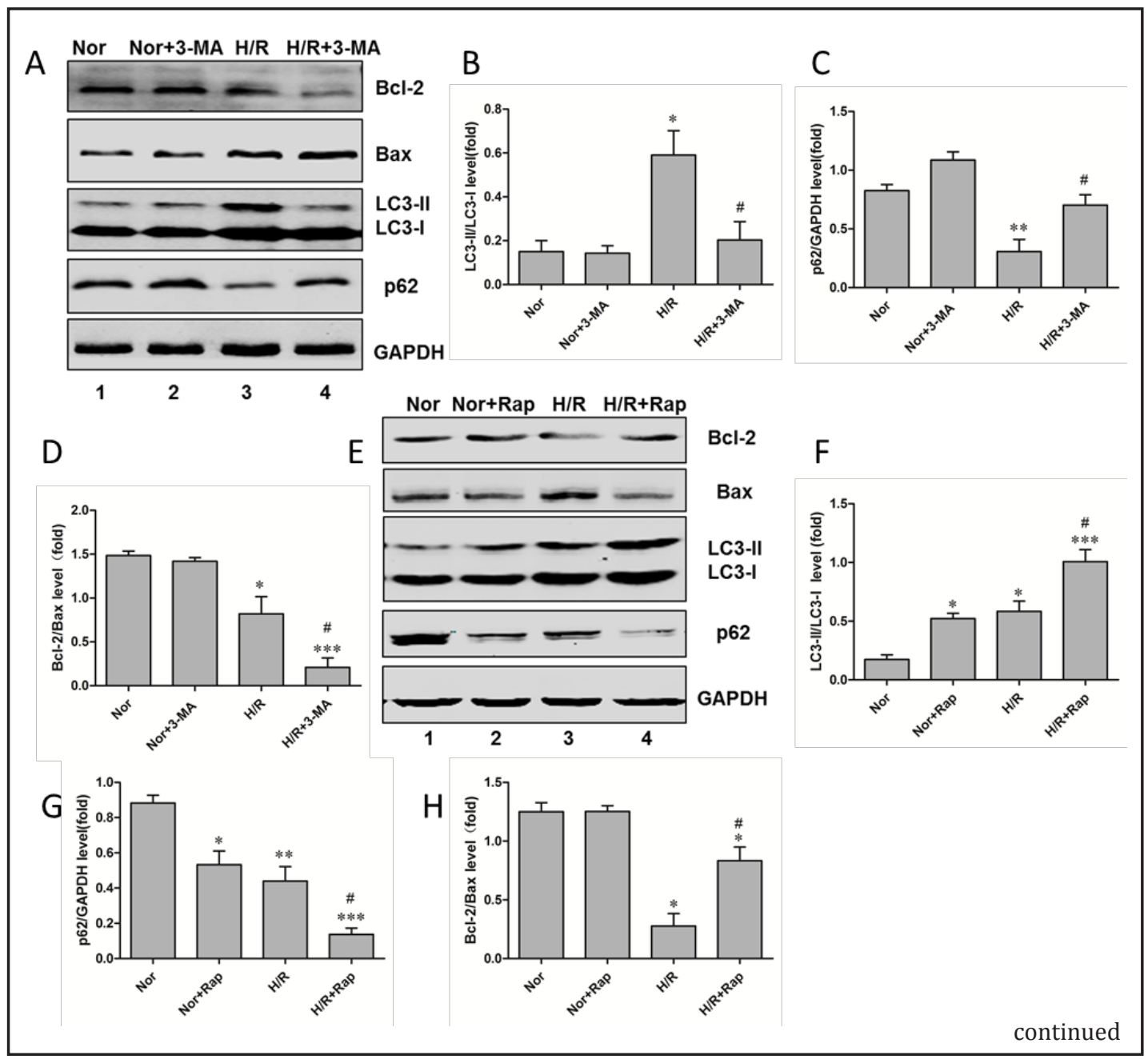

Fig. 5. Inhibition of autophagy by 3-MA or activation of autophagy by rapamycin aggravates or attenuates apoptosis in TCMK-1 in hypoxia/reoxygenation settings. 3-MA (5 mM) or rapamycin (1 uM) was administered before normoxia or 24-h hypoxia followed by 4-h reoxygenation. A-H: Representative immunoblots and densitometric analysis of LC3, p62, Bcl-2 and Bax in TCMK-1 lysates. The blots were reprobed for GAPDH. I and J: Representative images and numbers of TUNEL-staining cells $(\times 400)$. K-N: Representative immunoblots and densitometric analysis of p-mTOR, p-P70S6K and p-4E-BP1 in TCMK-1 lysates. The blots were reprobed for GAPDH. Data are expressed as mean \pm SD (for each group, $\mathrm{n}=3-10$ ). ${ }^{*} p<0.05,{ }^{* *} p<0.01$, ${ }^{* * *} p<0.001$ significantly different from normoxic group; ${ }^{\#} p<0.05,{ }^{\# \#} p<0.01,{ }^{\# \# \#} p<0.001$ significantly different from H/R group.

autophagy after 24-h exposure to hypoxia and subsequent 2-h reoxygenation was higher than that after 24-h exposure to hypoxia alone. However, after 4 and 8-h reoxygenation, autophagy began decreasing although was still higher than that in normoxic group. Finally, it declined to normal after 24-h reoxygenation, although the expression of p62 was still low at this time point, probably due to slow protein synthesis in vitro (Fig. 4, A-C). Electron microscopy showed that autophagosomes engulfed the cytoplasmic components when cells were exposed to 24-h hypoxia and subsequent 4-h reoxygenation, and some of cytoplasmic components were degraded (Fig. 4, D). Interestingly, immunoblot analysis and Annexin-V/ PI staining showed that apoptosis was not induced until hypoxia treatment for 24-h, which was aggravated more seriously in response to 4 and 8-h reoxygenation, and then decreased slightly after 24-h reoxygenation (Fig. 4, E-G). Consistent with cell apoptosis, the viability of 


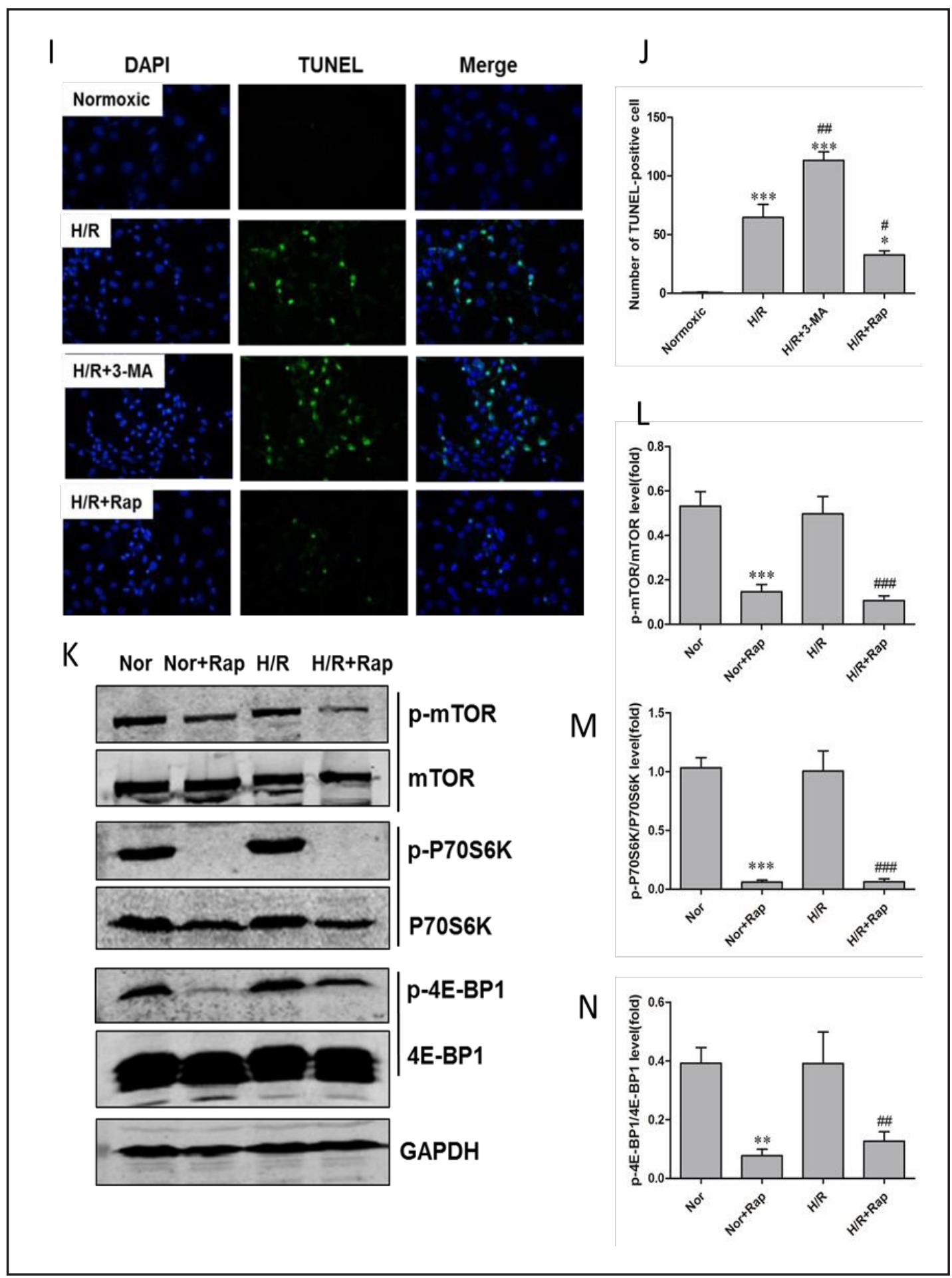

TCMK-1 declined drastically after 24-h exposure to hypoxia, and further worsened after 4 and 8-h reoxygenation. However, cell viability increased slightly when with reoxygenation was prolonged to 24-h, though it did not return to normal at this time point (Fig. 4, H).

The above findings suggest that autophagy was also an early response to hypoxia and further induced in the early stage of reoxygenation, which could partly slow down the increase of cell apoptosis, whereas the decrease of autophagy resulted in aggravation of apoptosis so that cell viability and apoptosis still did not return to normal until 24-h reoxygenation.

\section{KARGER}


Inhibition of autophagy by 3-MA or activation of autophagy by rapamycin aggravates or attenuates apoptosis in TCMK-1 in hypoxia/reoxygenation settings

We also examined the effect of autophagy in vitro in the presence of 3-MA or rapamycin on TCMK-1. Immunoblot analysis indicated that 3-MA pretreatment significantly decreased the level of autophagy in hypoxia/reoxygenation settings, as indicated by the decreased ratio of LC3-II/LC3-I (Fig. 5, A-B). Meanwhile, p62 degradation was inhibited so that the expression of p62 was obviously higher than that in H/R group (Fig. 5, A, C). In addition, cell apoptosis was aggravated in the presence of 3-MA during H/R injury, as evidenced by a lower ratio of Bcl-2/Bax (Fig. 5, A, D), which was further confirmed by the increased number of TUNEL-positive cells (Fig. 5, I-J).

In order to further confirm the protective role of autophagy, rapamycin was used to investigate whether induction of autophagy could alleviate cell injury. With addition of rapamycin prior to hypoxia, more LC3-1 was converted to LC3-II, and p62 degradation was accelerated as compared with H/R group (Fig. 5, E-G). With the change in autophagy, the ratio of Bcl-2/Bax increased significantly with rapamycin pretreatment in $H / R$, and morphological observations also showed the number of TUNEL-positive cells was reduced significantly (Fig. 5, E, H-J). The phosphorylations of mTOR and its downstream substrates P70S6K, 4E-BP1 were also inhibited significantly by rapamycin when TCMK-1 was incubated in normoxia or hypoxia/reoxygenation (Fig. 5, K-N). It is worth to mention that the dosage of 3-MA and rapamycin also did not induce apoptosis under normoxia, suggesting that induction of autophagy in response to hypoxia/reoxygenation played a cytoprotective role in $\mathrm{H} / \mathrm{R}$ settings.

\section{Discussion}

Despite the progress in the understanding about autophagy in kidney diseases [20-23], the time course of autophagy during renal I/R remains largely unclear. Whether autophagy contributes to cell survival or cell death also remains controversial [8, 22, 23]. In this study, autophagy was monitored in a time-dependent manner both in vivo renal I/R and in vitro $H / R$. It was found that autophagy was induced earlier than apoptosis so that it protected cells against damage. In addition, pharmacological agent 3-MA and rapamycin were added prior to ischemia and hypoxia, by which the renoprotection of autophagy was further clarified.

Autophagy is a conserved cellular catabolic process including five steps [24-26]: formation of phagophores (a double-membrane structure); elongation of phagophores; maturation of phagophores into autophagosomes that engulf intracellular components; fusion of autophagosomes with lysosomes forming autolysosomes; and degradation of the intracellular components. Monitoring the time course of autophagy and apoptosis especially during the early and recovery stage of renal I/R would help understand the relationship between them.

In the present study, we measured the autophagic activity through dynamic changes of LC3-II and p62 proteins [27, 28], and found that autophagy was induced earlier than apoptosis under renal I/R and cell hypoxia, which may postpone the occurrence of apoptosis or slow down the rate of apoptosis in the early stage of injury. Autophagy reached the maximum 2 days after reperfusion. At the same time, renal tissue apoptosis began declining slightly, and BUN and Scr also began decreasing significantly and nearly recovered to normal at day 3 of reperfusion, when autophagy was still higher than that in sham group. The reason that recovery of renal damage was earlier than that of autophagy might be due to engulfment of the damaged cytoplasmic components, resulting in the formation of autophagosomes and fusion with lysosomes to degrade, by which nutrients were recycled to improve repair of the kidney tissue. In in vitro $\mathrm{H} / \mathrm{R}$, autophagy was further induced after 2-h reoxygenation. However, with the time of exposure prolonging, autophagy decreased gradually. Meanwhile cell apoptosis reached the maximum and did not return to normal until 24-h reoxygenation. Based on these results, we supposed that decreased autophagy might weaken cellular 
clearance of damaged organelles and proteins, thus aggravating apoptosis and slowing down recovery from apoptosis after exposure to reoxygenation. In addition, the low level of p62 when autophagy was decreased after 24-h reoxygenation might be due to the lack of nutrient supply in vitro, thus weakening the ability of cells to synthesize protein. Above all, autophagy might play a renoprotective role in renal I/R and cell H/R.

To further confirm the renoprotective function of autophagy, 3-MA was administered prior to renal I/R and cell H/R. It was found that autophagy was suppressed significantly, and the renal tissue damage and tubular cell apoptosis were worsened. Knowing that autophagy is a double-edged sword and excessive activation of autophagy may cause tissue or cell damage, we therefore detected whether activation of autophagy by rapamycin would aggravate or alleviate renal tubular damage. It was found that rapamycin pretreatment increased autophagy markedly and partly mitigated the loss of renal function and apoptosis of renal tubular cells both in vivo and in vitro. Taken together, autophagy induced in renal I/R and cell H/R protected the kidney tissue and tubular cells against renal I/R injury in a timedependent manner, and up-regulation of autophagy could further ameliorate the injury.

Although some studies [29-31] reported that 3-MAat a concentration of $10 \mathrm{mM}$ may target other kinases and affect glycogen metabolism, lysosomal acidification and the mitochondrial permeability transition, numerous other studies showed that 3-MA at a concentration of $30 \mathrm{mg} / \mathrm{kg}$ [10] in vivo and $5 \mathrm{mM}[11,32]$ or $10 \mathrm{mM}[10,33]$ in vitro could inhibit autophagy effectively. It was found in our study that 3-MA was able to suppress autophagy effectively during renal I/R and cell $\mathrm{H} / \mathrm{R}$ without causing tissue and cell injuries in sham operation mice and TCMK-1 in normoxia. Also, there was no nephrotoxicity observed in sham operation group and normoxic group pretreated with rapamycin. It activated autophagy mildly without inducing excessive autophagy.

In 2010, Jiang et al. [10] provided evidence that hypoxia was able to induce autophagy in rat proximal tubular cells in vitro, suggesting that autophagy is an early response to hypoxic stress. In the present study, we monitored the time course of autophagy and apoptosis both in vivo renal I/R and in vitro $\mathrm{H} / \mathrm{R}$ and also found that autophagy postponed the occurrence of apoptosis as an early response to renal I/R. In addition, we used pharmacological agents to confirm the renoprotective function of autophagy. In theory, autophagy gene knockout represents a more specific approach than pharmacological agents to inhibit autophagy. Liu et al. [12] observed that deletion of tubular cell Atg5 dramatically sensitized the kidneys to I/R injury, suggesting that autophagy plays a role in maintaining tubular cell integrity under stress conditions. Thus, it is necessary to identify other Atg gene knockout mice and detect their role in renal I/R. Although we confirmed the renoprotective function of autophagy in renal I/R and TCMK-1 H/R, how autophagy protects renal tubular cells against injury remains elusive. Grahammer et al. [34] demonstrated that I/R injury exaggerated renal tubular damage in mTORC1-deficient mice. Evidence from other tissues or cell lines provided several signaling pathways that regulate autophagy during I/R or oxidative stress, including acetylated FOXO [33], AMPK/mTOR [35, 36], and HO-1/p38-MAPK [37, 38], but whether they involve in the renoprotection of autophagy during I/R needs to be further explored.

In summary, the results of our study on the time course of autophagy and apoptosis, and suppression and enhancement of autophagy not only confirmed the induction of autophagy but its renoprotective role in both in vivo renal I/R and in vitro H/R. Up-regulation of autophagy may prove to be a potential therapeutic target for the treatment of AKI.

\section{Acknowledgments}

This project was sponsored by the National Natural Science Foundation of China (No.81170687) and Shanghai Science and Technology Committee (No.134119a2300).

\section{Disclosure Statement}

None of the authors have any conflict of interest to declare. 


\section{Cellular Physiology Cell Physiol Biochem 2015;36:285-298 \begin{tabular}{l|l} 
and Biochemistry Published online: May 04, 2015 & $\begin{array}{l}\text { C 2015 S. Karger AG, Basel } \\
\text { www.karger.com/cpb }\end{array}$ \\
\hline
\end{tabular}

\section{References}

1 Oku M, Sakai Y: Peroxisomes as dynamic organelles: autophagic degradation. FEBS J 2010;277:3289-3294.

2 Corrochano S, Renna M, Tomas-Zapico C, Brown SD, Lucas JJ, Rubinsztein DC, Acevedo-Arozena A: $\alpha$-synuclein levels affect autophagosome numbers in vivo and modulate Huntington disease pathology. Autophagy 2012;8:431-432.

3 Cadwell K, Liu JY, Brown SL, Miyoshi H, Loh J, Lennerz JK, Kishi C, Kc W, Carrero JA, Hunt S, Stone CD, Brunt EM, Xavier RJ, Sleckman BP, Li E, Mizushima N, Stappenbeck TS, Virgin HW 4th: A key role for autophagy and the autophagy gene Atg16l/1 in mouse and human intestinal Paneth cells. Nature 2008;456:259-263.

4 Luciani A, Villella VR, Esposito S, Brunetti-Pierri N, Medina D, Settembre C, Gavina M, Pulze L, Giardino I, Pettoello-Mantovani M, D'Apolito M, Guido S, Masliah E, Spencer B, Quaratino S, Raia V, Ballabio A, Maiuri L: Defective CFTR induces aggresome formation and lung inflammation in cystic fibrosis through ROSmediated autophagy inhibition. Nat Cell Biol 2010;12:863-875.

$5 \quad$ He LY, Livingston MJ, Dong Z: Autophagy in acute kidney injury and repair. Nephron Clin Pract 2014;127:56-60.

6 Mizushima N: The pleiotropic role of autophagy: from protein metabolism to bactericide. Cell Death Different 2005;12:1535-1541.

7 Chen WQ, Sun YY, Liu KY, Sun XJ: Autophagy: a double-edged sword for neuronal survival after cerebral ischemia. Neural Regen Res 2014;9:1210-1216.

8 Suzuki C, Isaka Y, Takabatake Y, Tanaka H, Koike M, Shibata M, Uchiyama Y, Takahara S, Imai E: Participation of autophagy in renal ischemia/reperfusion injury. Biochem Biophys Res Comm 2008;368:100-106.

9 Kimura T, Takabatake Y, Takahashi A, Kaimori JY, Matsui I, Namba T, Kitamura H, Niimura F, Matsusaka T, Soga T, Rakugi H, Isaka Y: Autophagy Protects the Proximal Tubule from Degeneration and Acute Ischemic Injury. J Am Soc Nephrol 2011;22:902-913.

10 Jiang M, Liu K, Luo J, Dong Z: Autophagy Is a Renoprotective Mechanism During in Vitro Hypoxia and in Vivo Ischemia-Reperfusion Injury. Am J Pathol 2010;176:1181-1192.

11 Wang LT, Chen BL, Wu CT, Huang KH, Chiang CK, Hwa Liu S: Protective Role of AMP-Activated Protein Kinase-Evoked Autophagy on an In Vitro Model of Ischemia/Reperfusion Induced Renal Tubular Cell Injury. PLoS ONE 2013;8:1-15.

12 Liu S, Hartleben B, Kretz O, Wiech T, Igarashi P, Mizushima N, Walz G, Huber TB: Autophagy plays a critical role in kidney tubule maintenance, aging and ischemia-reperfusion injury. Autophagy 2012;8:826-837.

13 Jiang M, Wei Q, Dong G, Komatsu M, Su Y, Dong Z: Autophagy in proximal tubules protects against acute kidney injury. Kidney Int 2012;82:1271-1283.

14 Chien CT, Shyue SK, Lai MK: Bcl-xL augmentation potentially reduces ischemia/reperfusion induced proximal and distal tubular apoptosis and autophagy. Transplantation 2007;84:1183-1190.

15 Wu HH, Hsiao TY, Chien CT, Lai MK: Ischemic conditioning by short periods of reperfusion attenuates renal ischemia/reperfusion induced apoptosis and autophagy in the rat. J Biomed Sci 2009;16:1-10.

16 Tanida I: Autophagy basics. Microbiol Immunol 2011;55:1-11.

17 Ferraro E, Cecconi F: Autophagic and apoptotic response to stress signals in mammalian cells. Arch Biochem Biophys 2007;462:210-219.

18 Mizushima N, Yoshimori T, Levine B: Methods in Mammalian Autophagy Research. Cell 2010;140:313-326.

19 Ravikumar B, Vacher C, Berger Z, Davies JE, Luo S, Oroz LG, Scaravilli F, Easton DF, Duden R, O'Kane CJ, Rubinsztein DC: Inhibition of mTOR induces autophagy and reduces toxicity of polyglutamine expansions in fly and mouse models of Huntington disease. Nat Genet 2004;36:585-595.

20 Kume S, Uzu T, Maegawa H, Koya D: Autophagy: a novel therapeutic target for kidney diseases. Clin Exp Nephrol 2012;16:827-832.

21 Mizushima N, Komatsu M: Autophagy: Renovation of Cells and Tissues. Cell 2011;147:728-741.

22 Huber TB, Edelstein CL, Hartleben B, Inoki K, Jiang M, Koya D, Kume S, Lieberthal W, Pallet N, Quiroga A, Ravichandran K, Susztak K, Yoshida S, Dong Z: Emerging role of autophagy in kidney function, diseases and aging. Autophagy 2012;8:1009-1031.

23 Kaushal GP: Autophagy protects proximal tubular cells from injury and apoptosis. Kidney Int 2012;82:1250-1253.

24 Yang Z, Klionsky DJ: Eaten alive: a history of macroautophagy. Nat Cell Biol 2010;12:814-822. 


\section{Cellular Physiology Cell Physiol Biochem 2015;36:285-298

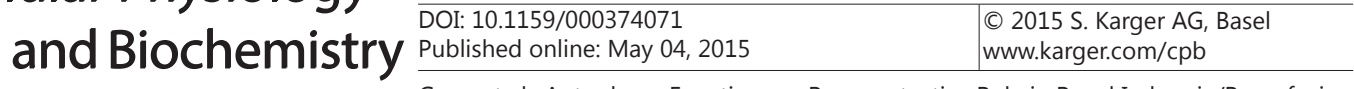 \\ Guan et al.: Autophagy Functions as Renoprotective Role in Renal Ischemia/Reperfusion}

25 Weide T, Huber TB: Implications of autophagy for glomerular aging and disease. Cell Tissue Res 2011;343:467-473.

26 Mizushima N, Levine B: Autophagy in mammalian development and differentiation. Nat Cell Biol 2010;12:823-830.

27 Yang J, Carra S, Zhu WG, Kampinga HH: The Regulation of the Autophagic Network and Its Implications for Human Disease. International Journal of Biological Sciences 2013;9:1121-1133.

28 Pankiv S, Clausen TH, Lamark T, Brech A, Bruun JA, Outzen H, Øvervatn A, Bjørkøy G, Johansen T: p62/ SQSTM1 binds directly to Atg8/LC3 to facilitate degradation of ubiquitinated protein aggregates by autophagy. J Biol Chem 2007;282:24131-24145.

29 Caro LH, Plomp PJ, Wolvetang EJ, Kerkhof C, Meijer AJ: 3-Methyladenine, an inhibitor of autophagy, has multiple effects on metabolism. Eur J Biochem 1988;175:325-329.

30 Punnonen EL, Marjomäki VS, Reunanen H: 3-Methyladenine inhibits transport from late endosomes to lysosomes in cultured rat and mouse fibroblasts. Eur J Cell Biol 1994;65:14-25.

31 Xue L, Borutaite V, Tolkovsky AM: Inhibition of mitochondrial permeability transition and release of cytochrome c by anti-apoptotic nucleoside analogues. Biochem Pharmacol 2002;64:441-449.

32 Zhao S, Luo H, Kan G, Zhao Y, Lin L, Tang Q, Yu C, Sun W, Cai L, Cui S: The Protective Role of Autophagy in Heterocephalus glaberHepatic Stellate Cells Exposed to H2O2 or Nutritional Stress. Cell Physiol Biochem 2014;34:463-473.

33 Han J, Pan XY, Xu Y, Xiao Y, An Y, Tie L, Pan Y, Li XJ: Curcumin induces autophagy to protect vascular endothelial cell survival from oxidative stress damage. Autophagy 2012;8:812-825.

34 Grahammer F, Haenisch N, Steinhardt F, Sander L, Roerden M, Arnold F, Cordts T, Wanner N, Reichardt W, Kerjaschki D, Ruegg MA, Hall MN, Moulin P, Busch H, Boerries M, Walz G, Artunc F, Huber TB: mTORC1 maintains renal tubular homeostasis and is essential in response to ischemic stress. Proc Natl Acad Sci USA 2014;111:E2817-26.

35 Chen BL, Wang LT, Huang KH, Wang CC, Chiang CK, Liu SH: Quercetin attenuates renal ischemia/ reperfusion injury via an activation of AMP-activated protein kinase-regulated autophagy pathway. J Nutr Biochem 2014;25:1226-1234.

36 Periyasamy-Thandavan S, Jiang M, Schoenlein P, Dong Z: Autophagy: molecular machinery, regulation, and implications for renal pathophysiology. Am J Physiol Renal Physiol 2009;297:F244-F256.

37 Wang Y, Shen J, Xiong X, Xu Y, Zhang H, Huang C, Tian Y, Jiao C, Wang X, Li X: Remote Ischemic Preconditioning Protects against Liver Ischemia-Reperfusion Injury via Heme Oxygenase-1- Induced Autophagy. PLOS ONE 2014;9:1-12.

38 Wang PR, Wang JS, Zhang C, Song XF, Tian N, Kong LY: Huang-Lian-Jie-Du-Decotion induced protective autophagy against the injury of cerebral ischemia/reperfusion via MAPK-mTOR signaling pathway. J Ethnopharmacol 2013;149:270-280. 